\title{
La mécanique des fluides à l'ordre de la vie ou Louis Vadot dans mon rétroviseur
}

\author{
Pierre Marion \\ Professeur Honoraire des Universités, Chirurgien honoraire des Hôpitaux de Lyon, La Tour Carrée, Chemin \\ de l'ancienne église, Poleymieux, 69250 Neuvilles/Saône
}

Nuit de Noël 1947. Il neige à New York. Nous retrouvons le vent glacial et la tempête à Gander, étape avant la traversée d'où partirent pendant la guerre tant de jeunes Américains pour atteindre la Grande-Bretagne et sauver l'occident menacé par les foudres hitlériennes. Dans ce Constellation je suis à l'aise : nous ne sommes que cinq voyageurs. J'essaie de revivre les moments essentiels de mes premiers contacts avec les médecins du nouveau monde. J'avais vu ce que je voulais voir. Essentiellement à Baltimore les moyens de blanchir les enfants bleus par une opération simple imaginée par A. BLALOCK et Helen TAUSSIG. A Boston, par D. HARKEN, j'avais appris la possibilité de dilater sans risques le rétrécissement de l'orifice mitral. Enfin, dans plusieurs laboratoires hospitaliers j'avais vu pratiquer l'exploration du cœur par cathétérisme, ce moyen qui allait faire progresser, de façon significative, le diagnostic et le traitement des maladies du cœur. Enfin, à Montréal, j'avais découvert la réanimation du cœur, en particulier, le traitement de la fibrillation des ventricules.

\section{L'exploration hémodynamique du cœur}

A New York, à l'hôpital Bellevue, j'avais vu A. Courrant, futur Nobel, et ses collaborateurs pratiquer le cathétérisme des cavités du cœur, technique réalisée déjà en 1929 sur lui-même par FORSSMAN, chirurgien allemand. Chauveau, à l'Ecole Vétérinaire de Lyon, les avait précédés et, dès 1860, démontré la faisabilité et l'innocuité de ce procédé sur le cheval, au moyen de sondes à ballonnets de fabrication artisanale.

La mesure des pressions dans les cavités cardiaques, celle du débit au repos et à l'effort allaient devenir une évaluation de routine. La connaissance de la mécanique du sang ouvrait le champ du diagnostic difficile des malformations congénitales et celui du degré d'insuffisance cardiaque. Avec le cathétérisme complété par l'angiocardiographie le chirurgien allait enfin pouvoir opérer avec la quasi certitude d'un diagnostic exact.

Les lois de la mécanique des fluides ont ouvert en chirurgie vasculaire la possibilité d'apprécier les différentes techniques d'anastomose, ainsi de comprendre les causes des succès ou des échecs. Dans ce chapitre Louis VADOT fut l'un des premiers à apprendre au chirurgien les caractères particuliers de la veine fluide à travers une anastomose termino-terminale, termino-latérale ou latérolatérale, et les causes des coagulations in situ.

Dans le domaine des anastomoses porto-cave, L. VADOT a proposé une règle simple basée sur la mesure des pres- sions veineuses en amont et en aval, en cours d'opération. Cette mesure est destinée à éviter les dangers de la suppression du débit de la veine porte dans le foie, source de troubles du comportement: encéphalopathie porto-cave. Ses calculs furent établis à l'aide d'une analogie électrique.

L'identité des expressions représentant la loi de Poiseuille d'une part et la loi d'Ohm d'autre part a incité L. VADOT à se servir d'une analogie électrique pour l'étude des circuits cardio-vasculaires.

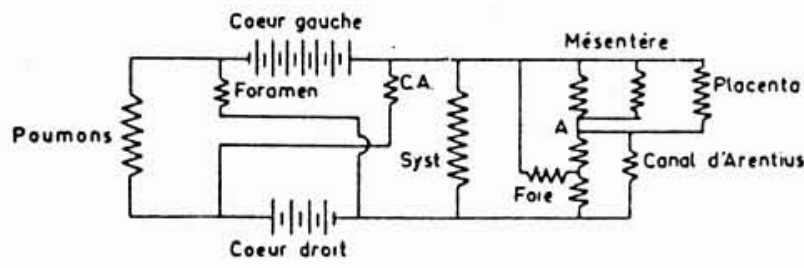

1. Schéma de la circulation fœtale. Exemple d'emploi d'une analogie électrique pour l'étude des circuits cardio-vasculaires.

La correspondance des trois éléments fondamentaux : pression vasculaire, débit sanguin, résistances vasculaires périphériques avec les valeurs électriques $V, I$ et $R$, suggère que la physiologie circulatoire et les situations pathologiques peuvent être analysées avec facilité dans des circuits électriques.

A cet effet, avec un appareil de sa conception (construit par Hellige-FranCE) L. VADOT a analysé avec précision et exactitude la plupart des circuits des cardiopathies congénitales, des lésions orificielles acquises du cœur, enfin les réseaux ramifiés vasculaires normaux et pathologiques. Sur une analogie électrique il est également possible de prévoir les conséquences d'un acte opératoire comme par exemple le shunt porto-cave.

Ce travail scientifique considérable, pont jeté entre la physiologie de Claude BERNARD et l'hydraulique, est résumé dans «La mécanique du cœur et des artères ». Ce chemin de recherche allait dans une bonne direction et l'hémodynamique "fille de l'hydraulique » s'est pliée à ses lois, à ses méthodes d'investigation et finalement a dégagé la cardiologie de l'ornière empirique. 


\section{La fibrillation des ventricules}

La crainte d'un arrêt brutal du cœur par l'accident de fibrillation des ventricules a longtemps freiné les tentatives d'opération directe sur le cœur de l'homme. La majorité des physiologistes croyait que ce phénomène était irréversible. Le Doyen de la Faculté de Médecine de Lyon, pour cette raison, me fit part de ses doutes sur l'avenir de la chirurgie du cœur et ceci avant mon départ pour les U.S.A. Heureusement pour moi, à Montréal, j'eus la chance d'une rencontre avec un chercheur et chirurgien MERCIER-FAUTEUX qui me fit découvrir la publication, datée de 1903, de deux physiologistes genevois Prévost et BATELLi qui avaient démontré l'efficacité des chocs électriques capables de bloquer la fibrillation et de faire cesser l'arrêt du cœur. Ce fut naturellement pour moi une grande satisfaction de découvrir ce moyen thérapeutique qui avait complètement échappé aux cardiologues et aux chirurgiens. Débarrassée de cette menace, la chirurgie cardiaque devenait possible. Aujourd'hui le défibrillateur est un instrument indispensable au cardiochirurgien et au réanimateur.

L. VADOT s'est intéressé au phénomène de fibrillation dans le cadre général de l'activité électrique du myocarde et du mécanisme de sa contraction. «Le myocarde formé d'un ensemble de cellules dont chacune constitue un réseau oscillant est un milieu électrique propre à la propagation de l'onde de l'excitation...

Dans la période d'activité rythmique normale, toutes les cellules sont synchronisées... la synchronisation est assurée par couplage transversal capacitif et ohmique... L'écroulement brutal de l'ordonnance cardiaque constitue le dramatique phénomène de fibrillation... Contraction cardiaque désordonnée, vermiculaire, inefficace qui aboutit à l'arrêt de la circulation. Il s'agit ici d'un phénomène analogue au décrochage de réseaux électriques initialement synchronisés. La cause de la fibrillation apparaît donc liée à une perturbation électrique des courants transversaux de synchronisation. »

\subsection{Place de la mécanique des fluides dans la chirurgie à cæur ouvert}

Grâce aux progrès dans le diagnostic, nombreux furent les malades qui ont bénéficié pendant les années 50 de l'opération de BLALOCK (dérivation d'une artère systémique dans l'artère pulmonaire), de la résection suture de l'aorte pour rétrécissement; enfin, de la dilatation digitale ou instrumentale à l'aveugle d'un rétrécissement mitral.

Toutefois les chirurgiens espéraient faire mieux. Leur ambition était d'opérer le cœur à sec pour le réparer. Dans ce but le programme théorique était :

- l'arrêt complet des circulations systémique et pulmonaire par des clampages vasculaires ad hoc;

- assumer artificiellement la circulation des organes et l'oxygénation du sang;

- assurer la reprise des mouvements cardiaques par la reprise de la circulation artificielle.

Ce programme, qui, à première vue, semblait à portée de main de l'ingénieur s'est heurté à de nombreuses difficultés consécutives à la fragilité particulière du fluide sanguin, véhiculé dans une circulation artificielle extra corporelle.
En 1950, à Paris, au Congrès International de Chirurgie, les résultats expérimentaux étaient de mauvaise qualité et l'on pouvait douter d'aboutir. C'est pourquoi un chemin nouveau, l'hypothermie, fut exploré.

\subsection{Chirurgie à cour ouvert en hypothermie légère (à $28^{\circ}$ - $30^{\circ}$ )}

La réduction de la consommation d'oxygène par les tissus à basse température selon la loi d'Arrhenius et Vant'off est connu de longue date. Dans cette voie deux travaux expérimentaux attirèrent l'attention des chirurgiens (BIGELOW \& Col. 1950, Canada ; Boerema, 1951, Amsterdam). En 1953, ce furent chez l'homme les premiers succès aux U.S.A. (Lewis et Taufic, Minneapolis; Swan et Virtue, Denver). Très rapidement la technique très simple qui consiste à placer sous anesthésie générale l'opéré dans un bain glacé fut adoptée. Le problème essentiel était celui de la connaissance de la durée admissible de l'arrêt circulatoire total suivant le degré de la température. Le cerveau étant l'organe le moins résistant à l'ischémie, le risque encouru par un arrêt circulatoire total était de provoquer des lésions cérébrales définitives.

Dans un travail, à la fois expérimental et clinique, Louis VADOT a établi des règles qui définissaient le délai admissible en fonction de la température, du poids et de l'âge du sujet opéré.

Un autre risque devait être aussi évité, l'arrêt du cœur par fibrillation des ventricules. L'expérience a prouvé que l'accident de fibrillation apparaissait dans la descente thermique à partir de $28^{\circ}$. Pour éviter ces accidents cérébraux et cardiaques et obtenir un bon délai de clampage, Louis VADOT a suggéré le refroidissement localisé autour du cou et de la tête. L'étude des courbes de l'hypothermie générale ainsi pratiquée montre qu'en un point la température cérébrale (mesurée dans le sang jugulaire) est à deux degrés au-dessous de celle du cœur. Ainsi, pratiquée à ce moment précis, la protection du cerveau est donc meilleure et le risque de fibrillation diminué.

Malgré la bénignité prouvée de cette méthode, l'hypothermie de surface a été abandonnée parce qu'elle ne donnait que 7 minutes et au plus 9 minutes à $28^{\circ}$ pour opérer. Lorsque la $7^{\mathrm{e}}$ minute fatidique était dépassée c'était le stress pour toute l'équipe chirurgicale. Ainsi l'hypothermie de surface ne fut qu'un moment de la chirurgie à cœur ouvert. Elle disparut lorsque la circulation extra corporelle fut enfin mise au point.

\subsection{La circulation extra corporelle (CEC)}

Aux USA furent obtenus les premiers succès de circulation extra corporelle chez l'homme. Après un cas isolé (GIBBON) en 1953, ce furent en 1954 les débuts de deux séries opératoires qui font date dans l'historique de la chirurgie à cœur ouvert : celle de C.W. LILLEHEI à Minneapolis puis de J. KIRKLIN à la Mayo Clinic, (Rochester, Min.).

L'appareil de la Mayo Clinic, très sophistiqué, partiellement automatisé, délivrait un débit sanguin voisin du débit normal chez l'homme. Les pompes étaient à galets. L'oxygénateur, innovation originale, était à grille. LILLEHEI avait fait précéder ses premières tentatives de CEC par des opérations à cœur ouvert sous circulation croisée.

Le receveur dont le poids est inférieur au donneur reçoit le sang du donneur pendant l'exclusion cardiaque. Une 
pompe à galets équilibre l'arrivée et la sortie du sang chez l'opéré. Huit opérations furent ainsi pratiquées pour des malformations congénitales sous circulation croisée entre le père ou la mère et leur enfant d'environ 20 kilos. Sans incident technique. Toutefois les risques encourus par le donneur s'opposaient à la continuité de ce moyen de traitement d'attente avant la CEC.

L'appareil de Minneapolis avait pour lui sa simplicité et sa reproductibilité :

- une pompe péristaltique de forme particulière (pompe à doigt de l'industrie laitière), pour assurer la circulation systémique ;

- pas de pompe d'aspiration, assurée par gravitation ; — un oxygénateur à bulles (DEWALL): le sang mélé à l'oxygène introduit à la base d'un tube vertical monte sous forme de bulles puis perd son trop plein d'oxygène dans un réservoir avant d'être repris par la pompe et propulsé dans la circulation.

Pour les chirurgiens et les techniciens ce fut un dur apprentissage.

Pendant une décade, cette chirurgie fut très meurtrière. Les troubles de la coagulation consécutifs au traumatisme du sang - globules, plaquettes - et à l'utilisation d'héparine pour éviter les coagulations dans les tuyaux furent parmi les accidents les plus fréquents. Leur gravité manifestée par des hémorragies ou a contrario par des thromboses furent l'opprobre de cette chirurgie.

Ainsi la voie était ouverte aux ingénieurs pour des recherches sur les éléments constitutifs de l'appareillage : les matériaux employés, les pompes, les oxygénateurs, les réservoirs, les formes de canules etc..., enfin, des recherches particulières sur la morphologie de l'échangeur thermique indispensable pour assurer l'équilibre thermique ou l'hypothermie pendant l'acte opératoire.

\subsection{La pompe S.O.G.R.E.A.H.}

Dès mon retour des U.S.A. en 1948 je me suis intéressé à la CEC, aux pompes à galets et l'oxygénateur de Jungbloed. Les progrès que nous avons pu réaliser dans ce domaine furent le résultat de recherches communes avec S.O.G.R.E.A.H. M. DANEL, grand patron, bouillonnant d'idées accepta de détacher dans mon équipe M. Louis VADOT qui a mis son talent au service de la chirurgie cardiaque soit au laboratoire soit en salle d'opération. La pompe proposée par Louis VADOT décrite dans la thèse de Jean-François EsTANOVE est une pompe à galets. L'innovation porte sur la forme du tuyau écrasé. En coupe le tuyau a une forme d'ellipse. De ce fait, l'écrasement par le galet est total, central et périphérique. Contrairement à ce qui est observé sur un tuyau cylindrique où l'écrasement laisse à la périphérie deux petites rigoles latérales, source de reflux. La pompe S.O.G.R.E.A.H. est occlusive, sans fuite rétrograde, et de ce fait peu hémolysante.

Présentée avec un tuyau de faible capacité elle peut facilement être adaptée aux enfants.

L'échangeur thermique proposé était à l'époque, dans les années 60, une innovation intéressante - échangeur à plaques avec un écoulement sans turbulence et un rendement excellent.

Lorsqu'apparurent les oxygénateurs à membrane Louis VADOT avait suggéré et réalisé, à titre expérimental, un oxygénateur capable par les mouvements de la membrane de faire progresser le sang et de réaliser ainsi une pompe oxygénateur.

En raison des incidents provoqués par la circulation artificielle en normothermie les cardio-chirurgiens eurent l'idée de combiner l'hypothermie profonde et la CEC, dans le but de réduire les besoins de l'organisme et de ce fait de diminuer les débits et même d'arrêter totalement la circulation pendant le temps d'ouverture du cœur.

Avec la pompe S.O.G.R.E.A.H. nous avons ainsi pratiqué cette chirurgie en hypothermie profonde soit avec un oxygénateur soit en utilisant pendant le refroidissement et le réchauffement les poumons du patient (technique de Drew, Londres).

Pendant le temps de l'arrêt circulatoire total la correction cardiaque était réalisée. D’après les calculs théoriques la durée admissible de l'arrêt circulatoire total à basse température $\left(10^{\circ}\right.$ et $\left.15^{\circ}\right)$ était de l'ordre de 45 minutes. Au-delà d'une heure des troubles cérébraux ischémiques étaient à craindre.

Heureusement, peu à peu, les améliorations techniques de l'appareillage, l'emploi du sang dilué ont fait régresser les troubles consécutifs au traumatisme sanguin et finalement la CEC a été pratiquée à débit réduit et l'hypothermie cardiaque profonde provoquée par action locale sur le cœur. C'est ainsi que la correction des lésions congénitales ou acquises du cœur est actuellement pratiquée. En particulier le remplacement des orifices du cœur par une prothèse.

\subsection{Les prothèses valvulaires cardiaques}

C'est en 1960, aux U.S.A., que furent mises en place, avec succès, les premières prothèses valvulaires (l'orifice aortique par D. HARKEN et l'orifice mitral par STARR). Les premières prothèses valvulaires étaient à bille, en matière plastique, maintenues dans une cage métallique. Vieux procédé inspiré d'un brevet industriel U.S.A. datant de 1858 pour la fermeture des bouteilles d'eau gazeuse.

Les prothèses à bille fabriquées par STARR et EDWARDS, 30 ans après leur naissance, sont encore souvent utilisées.

Les prothèses à disque basculant ont succédé aux prothèses à bille sous de multiples formes et matériaux : disque basculant autour d'un axe ou retenu par des griffes, disque horizontal maintenu dans une cage métallique. Les plus employées furent créés par LILLEHEI-KASTER et par BjoRK (fig. 2). La dernière prothèse diffusée, la plus employée, est une bivalve en forme d'ailes de papillon (St. Jude), à ouverture latérale.

A cette grande variété morphologique des prothèses mécaniques, il faut ajouter les prothèses biologiques, d'origine humaine prélevées sur le cadavre et surtout d'origine animale - Valves de porc conservées dans une solution de glutaraldéhyde (A. CARPENTIER) qui a pour résultat de dépersonnaliser les tissus prélevés, de transformer une greffe tissulaire en une simple prothèse biologique, afin d'éliminer les réactions de rejet.

\subsection{Essais sur modèles hydrauliques}

On sait, maintenant, apprécier la qualité hémodynamique d'une prothèse par une évaluation sur des modèles hydrauliques mécaniques. Dans ces appareils on fait passer un fluide dont la viscosité est voisine de celle du sang. La 

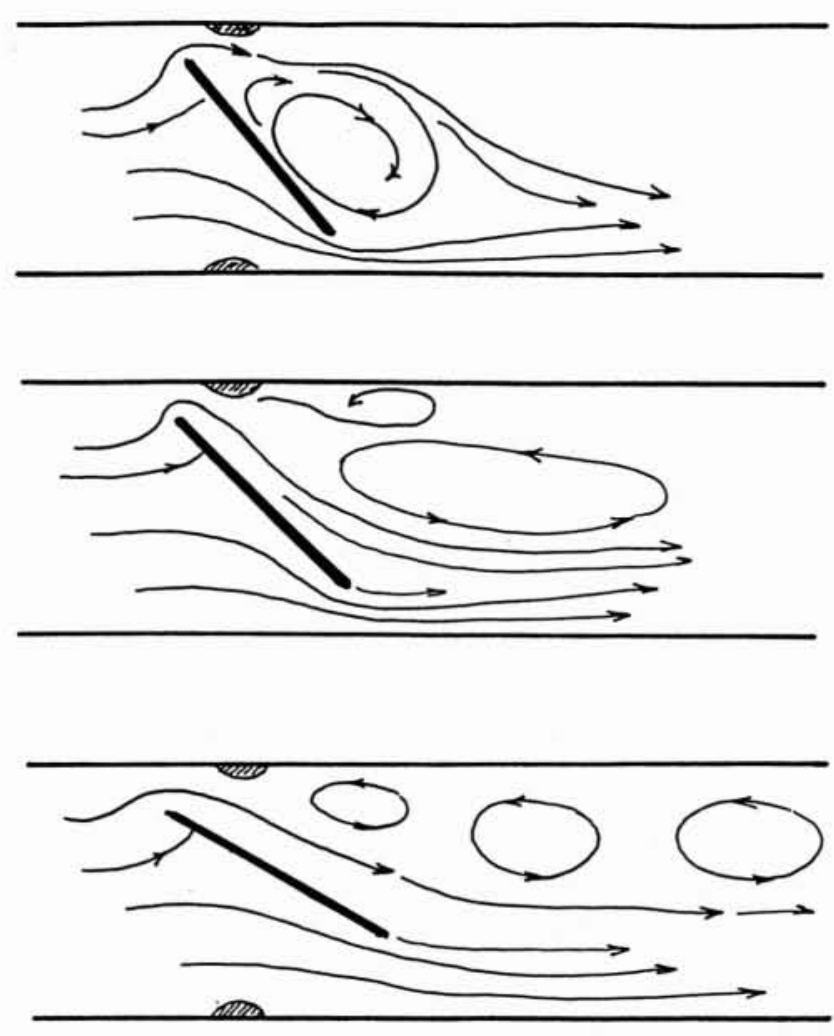

2. Mono-valve.

Début d'ouverture: Gros tourbillon lié à la valve. Zone de fort cisaillement le long de la ligne de jet.

Demi-ouverture : Recolement sur l'extrados de la valve. Gros tourbillon de sillage.

Pleine ouverture. Veine principale déviée sur un côté. La section utile réduite à moins de la moitié de la section du vaisseau. Zone de turbulence persistante.

circulation est soit à débit continu soit pulsatile. La prothèse étudiée est placée à la sortie du ventricule artificiel comme l'orifice aortique normal. Le fluide qui la traverse est ensuite récupéré et renvoyé dans la pompe et ainsi de suite.

Par des moyens assez simples (mesure électronique des pressions en amont en en aval, des débits, observation photographique ou cinématographique de l'écoulement, mesure de la vitesse des écoulements dans la veine fluide) il est possible d'évaluer d'une façon précise la qualité d'une prothèse valvulaire. Dans ce but, Louis VADOT a construit pour notre équipe plusieurs modèles qui, plus tard, nous ont servi pour l'évaluation des cœurs artificiels (figs. 3 et 4). Les tests visent à apprécier, surtout, les points suivants :

- les pertes de charge provoquées et leur courbe en fonction de l'augmentation du débit,

- le volume de la fuite : fuite active pendant la fermeture et fuite passive, prothèse fermée,

- les turbulences intra-valvulaires, les points d'accélération et de ralentissement,

— la fiabilité à long terme.

Les résultats globaux des évaluations conduisent à des critiques dans le fonctionnement des valves artificielles actuellement employées.
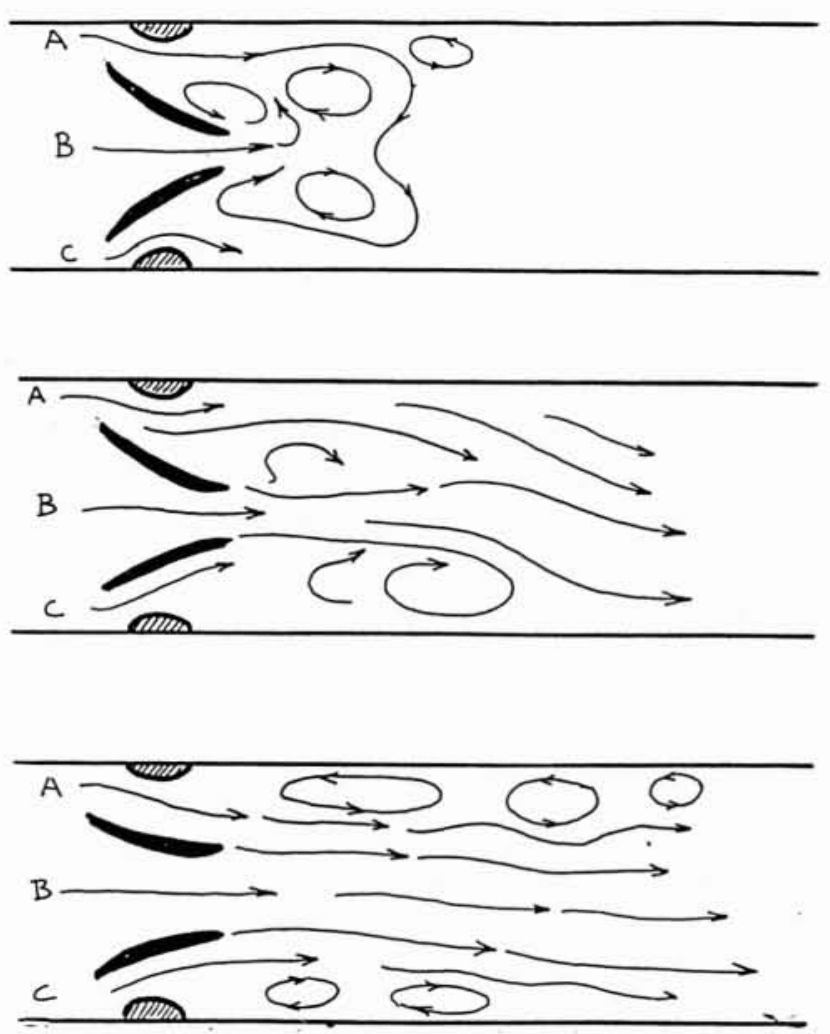

3. Bi-valve à ouverture centrale.

Début d'ouverture: Système tourbillonnaire transitoire induit par la veine latérale $A$ et la veine centrale $B$.

Demi-ouverture : La veine centrale B et la veine latérale, induisent un système tourbillonnaire transitoire complexe siège de turbulence. Par suite de la prépondérance de veine $A$. L'ensemble est dévié du côté opposé. Pleine ouverture : La veine centrale et les veines latérale créent un courant central bien ordré dont la section efficace est de l'ordre de $2 / 3$ à $3 / 4$ de la section du vaisseau, mais des turbulences latérales subsistent.

Toutes les prothèses de petit calibre entraînent une perte de charge considérable.

Toutes les prothèses, mais surtout les prothèses mécaniques, sont responsables d'une fuite diastolique (surtout à la fermeture) que l'on peut évaluer entre $15 \%$ et $30 \%$ du volume systolique éjecté.

Les prothèses mécaniques sont fiables à long terme, les biologiques ne le sont pas (vie courte chez les enfants et chez l'adulte : calcifications, fractures fréquentes à partir de 7 ans d'implantation). Les thromboses locales et leurs complications sont plus fréquentes avec les prothèses mécaniques qu'avec les prothèses biologiques.

Tout compte fait, la mortalité tardive après dix ans, par accident, relevant de la prothèse n'est pas négligeable. Néanmoins, la durée de vie, et c'est là l'essentiel, d'un porteur de prothèse est plus longue que l'évolution naturelle de la maladie naguère observée. Environ $70 \%$ des malades survivent 10 ans après l'implantation d'une prothèse. Ainsi s'explique que des milliers de malades acceptent dans le monde le remplacement valvulaire. Mais ces succès ne doivent pas laisser dans l'ombre les échecs dont certains relèvent d'imperfections dans la forme ou le matériau employé. 

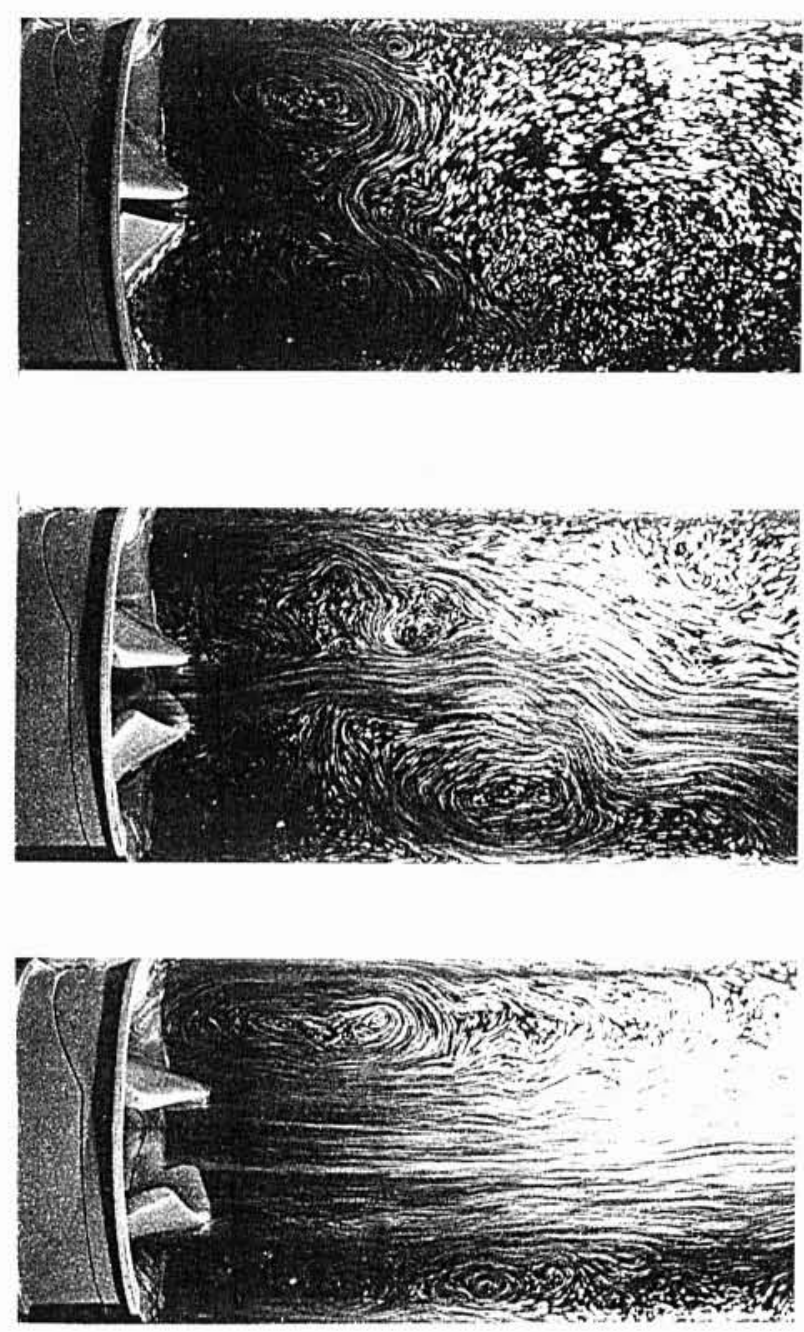

4. Ecoulement sur modèle à bi-valve à ouverture centrale.

\subsection{Recherches}

Les recherches dans ce domaine sont donc parfaitement justifiées. Elles se font dans trois directions :

- a) Prothèses biologiques — recherche de l'augmentation de la durée de vie.

— b) Prothèses mécaniques - essai d'amélioration de la valeur hémodynamique et de la qualité du matériau.

A cet égard les travaux de D. LAPEYRE et M. DASSAut ont abouti à une innovation intéressante - une prothèse tri-valvulaire avec des volets maintenus par de miniscules crochets, un très bon profil d'écoulement. Sur les bancs d'essai cette nouvelle prothèse à ouverture centrale possède de très bonnes qualités hémodynamiques. Et les premiers essais expérimentaux sont satisfaisants.

- c) Prothèses mécaniques assistées - De sérieux arguments plaident en faveur de la recherche d'un moyen pour assister la fermeture d'une prothèse mécanique.

Arguments expérimentaux : Le reflux, à chaque diastole, en rythme normal, se situe entre $10 \%$ et $20 \%$ du volume systolique, entraînant une fatigue du ventricule; les prothèses sollicitées à un rythme rapide ne se ferment plus, perdent leur étanchéité et provoquent l'arrêt circulatoire.

Arguments cliniques: La mort subite chez les porteurs de valves atteint dans la majorité des statistiques $15 \%$ à
$20 \%$ de la mortalité globale. On peut supposer que cet accident est la conséquence directe ou indirecte (thrombose) d'une accélération anormale et brutale du cœur (tachycardie, tachyarythmie) qui entraîne la non-fermeture de la prothèse. Partant de ces faits nous avons Jean CoLoN et moi, proposé la fermeture ou l'ouverture des volets par l'action d'un couple de torsion.

Dans un volet, un fil élastique pré-contraint est fixé d'une part au volet et d'autre part à l'anneau. En position d'ouverture l'élasticité du fil ramène le volet en position de fermeture. Ce dispositif peut être placé sur un ou plusieurs volets. Les premiers tests hémodynamiques sur une prothèse à deux volets plats qui s'ouvre à $90 \%$ sont favorables : disparition de la fuite active pendant la fermeture et faible perte de charge malgré une certaine résistance à l'ouverture. Il est évident que le choix du fil conditionne la fiabilité du dispositif et que de nouvelles recherches sont nécessaires.

La fermeture d'une prothèse à volet peut également être obtenue par action magnétique (A. CARPENTIER) mais le couple de torsion me parait préférable à l'action magnétique pour la simple raison que l'attraction exercée par l'élasticité du fil se fait surtout en période d'ouverture maximale, ce qui n'est pas le cas pour l'attraction magnétique.

Quoiqu'il en soit du procédé, le principe de l'assistance active d'une prothèse mérite des recherches nouvelles, en particulier dans le but de la réalisation du cœur artificiel.

\section{De la pompe à sang au cœur artificiel}

\subsection{Premières tentatives}

Depuis William HARveY, on considère le cœur comme une pompe ou plutôt comme deux pompes disposées en série. L'une assure la circulation générale dite systémique et l'autre la circulation pulmonaire où se fait l'oxygénation du sang. L'idée de remplacer ces deux pompes naturelles par deux pompes artificielles est donc logique et a priori les difficultés ne paraissent pas insurmontables. En réalité l'apparente facilité cache bien des difficultés. En témoignent les multiples tentatives qui jalonnent l'historique du cœur artificiel depuis 40 ans. Dès les premiers essais furent adoptées deux pompes volumétriques à membrane reliées à l'extérieur du thorax à une source d'énergie pneumatique. L'équilibre entre la pompe droite et la pompe gauche était assuré par un asservissement réglé sur la fréquence, les pressions et les débits.

1953 - Zacouto, Paris : Premiers essais expérimentaux connus (thèse 1958).

1958 - Akutzu et Kolf : Premiers résultats expérimentaux aux USA.

1962 - Akutzu : Survie d'un veau - 24 h.

1969 - D. Cooley et D. LiotTA : Première tentative chez l'homme - Survie : $64 \mathrm{~h}$, avant une greffe qui fut un échec.

1973 - Akutzu : Survie d'un veau pendant 10 jours.

1985-1993 - Appareils de Jarvick, de Pierce, de Copeland et finalement les premières applications d'un cœur artificiel animé par une source d'énergie électromagnétique extra corporelle.

Les pionniers du cœur artificiel avaient espéré que le but serait atteint par étapes :

- La première étape : le cœur est remplacé par une pompe intra corporelle avec un moteur et une source d'énergie en position extra corporelle. 
— La deuxième étape : les pompes et l'activateur en situation intra corporelle, la source énergétique est en position extra corporelle.

— La troisième étape : tous les éléments, pompes, activateur, source d'énergie sont implantés dans le thorax.

\subsection{Où en est-on aujourd'hui?}

La première étape a été franchie chez l'homme, généralement dans l'attente d'une greffe. La deuxième étape commence. Plusieurs fois le cœur artificiel et son moteur ont été implantés dans le thorax reliés à une source d'énergie électrique extra corporelle. Ce dispositif est relié par fils transcutanés au moteur. La dernière formule employée, la moins contraignante pour le malade, consiste à utiliser une source électromagnétique externe dont l'action sur le moteur se fait par voie per cutanée. Ainsi, à Houston, M. Templeton, qui ne pouvait recevoir une greffe a survécu un an mais il est mort d'une complication cérébrale. Dans ce cas particulier et dans d'autres analogues il ne s'agissait pas de remplacement total du cœur mais d'une assistance au ventricule gauche par une pompe intra thoracique avec son activateur (formule préconisée, il y a plusieurs années, à Houston, par COOLEY et NORMAN et qui a fait l'objet de travaux expérimentaux à Grenoble par le professeur LATREILLE et son équipe. Un long chemin reste donc à parcourir avant la diffusion et la fabrication industrielle. Néanmoins, on espère que se développeront rapidement les procédés analogues à ceux qui ont été employés aux USA.

\subsection{Quelle énergie implantable pour le futur?}

Il n'est pas possible de prévoir actuellement l'implantation de l'énergie dans le thorax. En effet: l'énergie atomique serait évidemment capable d'assurer ce rôle - cela a été démontré par la NASA - mais la multiplication des implantations de ce type entraînerait de grands risques pour la société. La commission américaine Atomic Energy en a déconseillé formellement l'application.

Plusieurs hypothèses de travail s'ouvrent à la recherche, aucune n'est satisfaisante. L. VADOT a retenu comme possible une production d'énergie implantée à partir d'échanges calorifiques organiques. L'énergie serait trouvée dans les différences de température existant entre les organes. En pratique, entre le point le plus chaud de l'organisme, le foie à $37,5^{\circ}$ en moyenne, et le point le plus froid, les tissus sous cutanés $25^{\circ}$, un circuit themique fermé (gaz fréon, amoniac) emprunterait la chaleur au foie et l'évacuerait sous la peau. Dispositif, on le comprend, analogue à celui relevant de l'utilisation de l'énergie thermique des mers.

\subsection{Caur artificiel monocoque}

Dans le but d'assurer facilement la régulation entre les deux pompes à membranes droite et gauche et l'équilibre entre les deux circulations, l'idée nous est venue de relier les deux pompes par un shunt gauche/droit unidirectionnel placé entre les oreillettes ou les ventricules. Ainsi une seule source d'énergie suffit pour alimenter les deux pompes jumelées comme dans un cœur normal.

Avec une CIV, Didier LAPEYRE a fait survivre un mouton pendant 3 jours. Mais pour des raisons de financement les recherches n'ont pas pu être poursuivies à Lyon, en particulier l'intérêt d'une communication unidirection- nelle, gauche/droite, entre les oreillettes envisagée par L. VADOT. Poursuivant l'idée de la réalisation d'un cœur monocoque D. LAPEYRE, en collaboration avec l'Aerospatiale, a conçu un modèle sans shunt, activé par une seule source d'énergie alimentant les deux chambres ventriculaires sous deux pressions différentes. Ce modèle de forme anatomique a l'avantage d'être peu encombrant. Il est conçu pour être placé d'urgence.

\section{En manière de conclusion}

De ce rapide survol de la chirurgie cardiaque on retiendra que de nombreuses voies de recherche restent ouvertes pour l'ingénieur hydraulicien. Quelques suggestions dans des registres bien différents :

- Pompes à sang. Prothèses.

- Valeur des pompes rotatives à grande vitesse.

- Perfectionnement des moyens d'énergie électromagnétique externe.

- Energie implantable.

- Nouveaux procédés de mesure hémodynamique, non invasifs.

- Instrumentation vasculaire pour réaliser par voie per cutanée la désobstruction des artères, en particulier des coronaires, etc...

Cette liste n'est pas exhaustive...

Ces suggestions induites par 25 ans de collaboration sans nuage, sans contrat notarié avec L. VADOT, me conduit naturellement une nouvelle fois à fouiller dans mon rétroviseur.

Nous sommes en 1957, dans une grande brasserie lyonnaise : je revois, M. DANEL, PDG de la SOGREAH. En l'écoutant parler d'avenir, de recherches et de maquette hydrauliques, de futures conquêtes industrielles, j’imagine que ses services de recherche, capables de construire les plus grands barrages du monde, de créer les turbines de l'usine maréomotrice de la Rance trouveront la solution au problème de la CEC puis du cœur artificiel. La suite des événements a fait la preuve que je ne m'étais pas trompé. Merci, mon Cher ami, L. VADOT. Merci d'avoir fait progresser la chirurgie cardiaque et bénéficier les opérés de votre science, de vos talents et de votre générosité.

\section{Bibliographie}

EstanOve J.F. - Hypothermie profonde - Technique hémodynamique. (Expansion Scientifique Française, collection P. Santy).

LAPEYRE D. - a Points critiques qui restent à résoudre pour que le caur artificiel puisse entrer dans l'arsenal thérapeutique de routine ». Annales de Chirurgie - 44/2/1990, pp. 182-183.

MARION P. - Afin que batte le caur - Epopée de la chirurgie cardiaque. (Presse Universitaire de Lyon, 1990).

Marion P., Colon J., VAdot L. - « Prothèse mitrale assistée ». (Annales de Chirurgie - 44/1990, pp. 161-162).

VAdot L. - Hypothermie - De la Physique à la Physiopathologie per opératoire. (Expansion. Ed. 1962, collection Paul Santy).

VADOT L. - Mécanique du caur et des artères. (Expansion Ed. Collection P. Santy, 1967).

WATSON J.L., Domanski M.J., Lenfant C. - «Suppléant : circulatoire mécanique pour défaillance cardiaque : Perspective à long terme. (Annales de Chirurgie - 44/2/1990, pp. 163-167). 